\title{
Moving Toward Remote, Parent-Reported Measurements in Pediatric Anthropometrics for Research and Practice
}

\author{
Eliot N. Haddad ${ }^{1}$, Tsoline Kojaoghlanian ${ }^{2}$ and Sarah S. Comstock ${ }^{1 *}$ \\ ${ }^{1}$ Department of Food Science and Human Nutrition, Michigan State University, East Lansing, MI, United States, \\ ${ }^{2}$ Department of Pediatrics, Maimonides Children's Hospital, Brooklyn, NY, United States
}

Keywords: pediatrics, anthropometry, remote measurement, telemedicine, parental report

\section{INTRODUCTION}

Anthropometry is the study of human anatomical measurements and can provide insight into growth, energy/nutritional intake, physical fitness, and clinical care (1). Common anthropometric measurements include body/head circumferences, height, weight, and skinfold thickness. Such anthropometric measurements underlie further proxies for health/growth such as body mass index (BMI).

The accurate attainment of body measurements and proportions is especially relevant to the field of pediatrics, where growth occurs rapidly and must be quantified in reliable and valid fashions. Anthropometry in pediatrics aids in the diagnosis of such conditions as stunting, wasting, congenital/acquired hormonal disturbances, brain development, and malnutrition (1). For example, the mid-upper arm circumference allows for insight into the nutritional status of a child or a pregnant woman. Similarly, length/height-for-age, weight-for-age, weight-for-length/height, and BMI-for-age are all useful as growth indicators in pediatric populations. These metrics are best reported as z-scores (number of standard deviations away from a reference median/mean) due to their ease of interpretation and ability to be statistically summarized (mean, median, quartiles) (2). In research, these data can be applied in epidemiological models to identify potential associations that can inform public policy to address population-level nutritional or lifestyle deficits. Clinically, anthropometric measurements directly inform diagnosis and assessment. Herein, we discuss how to approach remote pediatric anthropometry for research and healthcare during a pandemic, where physical engagement is limited.

This article was submitted to

Pediatric Gastroenterology, Hepatology and Nutrition,

a section of the journal

Frontiers in Pediatrics

Received: 18 December 2021

Accepted: 14 February 2022

Published: 08 March 2022

Citation:

Haddad EN, Kojaoghlanian T and Comstock SS (2022) Moving Toward

Remote, Parent-Reported

Measurements in Pediatric

Anthropometrics for Research and

Practice. Front. Pediatr. 10:838815.

doi: 10.3389/fped.2022.838815

\section{THE RISE OF REMOTE ANTHROPOMETRY}

Anthropometric data can be collected with the use of different tools and methods depending on the body site in question. For height and weight, a tape measure and scale are normally sufficient to obtain workable measurements, though a stadiometer is the ideal instrument for measuring height (3). In pediatric populations, especially those under 2 years, it is the length, rather than height, that is measured. Length measures in infants are often challenging to accurately obtain since the child must lie in a supine position for the duration of the measurement, which usually necessitates the help of an additional person. Additionally, skin folds and body circumferences are often more difficult to obtain due to their reliance on more intricate tools and nuanced procedures. For example, it may be unclear at which locations to measure circumferences and how to correctly use a caliper for an untrained individual. Due to this, a specialist is usually required for the attainment of anthropometric data other than height and weight. 
Though in-house clinical measurements by an anthropometric specialist are regarded as the gold standard of anthropometry (3), remote methods for height/weight measurement are becoming increasingly popular due to their recognized benefits of cost-efficiency on the part of researchers and participants alike, especially in the wake of the COVID-19 pandemic (4). For adults, several studies have validated selfreported height and weight measurements (5). However, in pediatric populations, parental self-reporting of anthropometrics is more prone to error due to the inherent difficulties of conducting the necessary procedures on a child or infant (2).

A retrospective study conducted during the current COVID19 pandemic in a single pediatric endocrinology center demonstrated that remote measurements among a cohort ranging from 3 to 18 years could be clinically useful due to their overall good concordance with in-person measurements (6). Interestingly, remote, parent-provided measurements of overweight and obese children were deflated for height. For all participants, weight tended to be deflated when reported by parents. This phenomenon has been demonstrated in other studies as well and seems to increase with child age (7-9), perhaps due to social desirability bias, lack of parent knowledge regarding proper measurement procedures, or imprecise scales. Nonetheless, with proper guidance, education, and adherence to best practice instructions, remote collection of pediatric anthropometric data is bound to become not just more commonplace, but also relied upon in clinical decision-making, especially in light of infectious disease pandemics that have forced ongoing health checks to transition to remote formats (4).

\section{LACK OF VALIDATION IN INFANT POPULATIONS}

Remote pediatric anthropometrics are typically reported by a parent/guardian of the infant or child and may be obtained in many ways. The first method of remote anthropometric measurement entails guiding a parent/caregiver in the process of measuring their child's body. This may occur live via videoconference and/or through an instructional pamphlet, available in multiple languages, that provides adequate detail for the parent to obtain measurements effectively and accurately. Such guidance has been shown to significantly improve parental measurements and increase the accuracy of pediatric BMI categorization based on remote measurements (10). This guided method of measurement has relevance for both research and clinical practice, though this assumes that the parent will have access to the necessary tools to collect such measurements. Indeed, no degree of guidance can overcome an absence of equipment, which is yet another consideration to be made when utilizing remote anthropometry. A parent may report pediatric anthropometrics based on an estimate or an unguided home measurement, but this is prone to much error and is unlikely to provide constructive information for clinical use (11).

In research studies, remote anthropometric data can be collected by simply taking measures from the child's medical records. Parental report based on recall from the last clinical visit is also a viable option. However, this may introduce recall bias or cause the reported measurements to be outdated depending on when the last visit occurred. This is especially true for infants, who grow swiftly in the first year of life. Though not yet widespread, there are also several technologies being developed that can allow for the collection of anthropometrics by way of three-dimensional scanning in mobile clinical/research settings (12). These novel methods synergize with telematics by allowing measurement data to be directly logged and transferred to physicians or researchers.

For infants especially, there are physical challenges involved in collecting measurements, even in a professional setting (2). Weight measurements for children under 2 years of age are typically collected using baby scales that provide a platform on which the infant can be set down. Occasionally, for infants who are particularly squirmy or agitated during the weighing procedure, a parent is weighed while holding the infant, then the parental weight is subtracted from that measure to obtain the infant weight. For length, infants are classically measured while recumbent on a specialized board that has a fixed headpiece and mobile footpiece. Through all this, infants often cry and kick, sometimes necessitating two persons to obtain accurate data. Hence, obtaining remote measurements for infants is particularly challenging, especially considering the specialized equipment that is usually necessary. Even in clinical settings, it is not uncommon for a documented measurement to be corrected during the same visit after the care provider plots the value in the infant's growth chart, realizes that the growth curve has an unexpected shape, and decides to take a second measurement. Older children can be measured for height and weight relatively easier than infants, since household scales and measuring tapes will ordinarily be sufficient for accurate measurements, though there is the possibility of systematic or random errors arising from the equipment or measuring techniques $(6,13)$. If these household tools are applied to infants for anthropometric measurements, the errors may further compound. Moreover, if a child or infant has a disease that impacts their motor function or attention span, measurement may be greatly hindered, especially for those parents who are not trained in the proper measurement procedures. Parents are deeply invested in their infant's growth, often citing actual numbers for weight and length to friends and family as a proxy for their child's health $(14,15)$. Thus, it is expected that parental interest and participation in learning and utilizing remote methods will be high. However, this may concurrently lead to social desirability confounding in the reported measures if faster growth is perceived as favorable by the parents and their social circles (16). Hence, validation of parent-reported values of infant anthropometric measurements is imperative considering the likelihood that remote methods will continue to proliferate in pediatric research as well as practice and given their potential to shed light on growth in geographically dispersed and medically underserved populations.

Because of these described challenges posed by pediatric anthropometrics, parental reports have not always been concordant with measurements obtained by a specialist (4). However, in envisioned clinical practice in the future and for large research studies, parental report is often the 


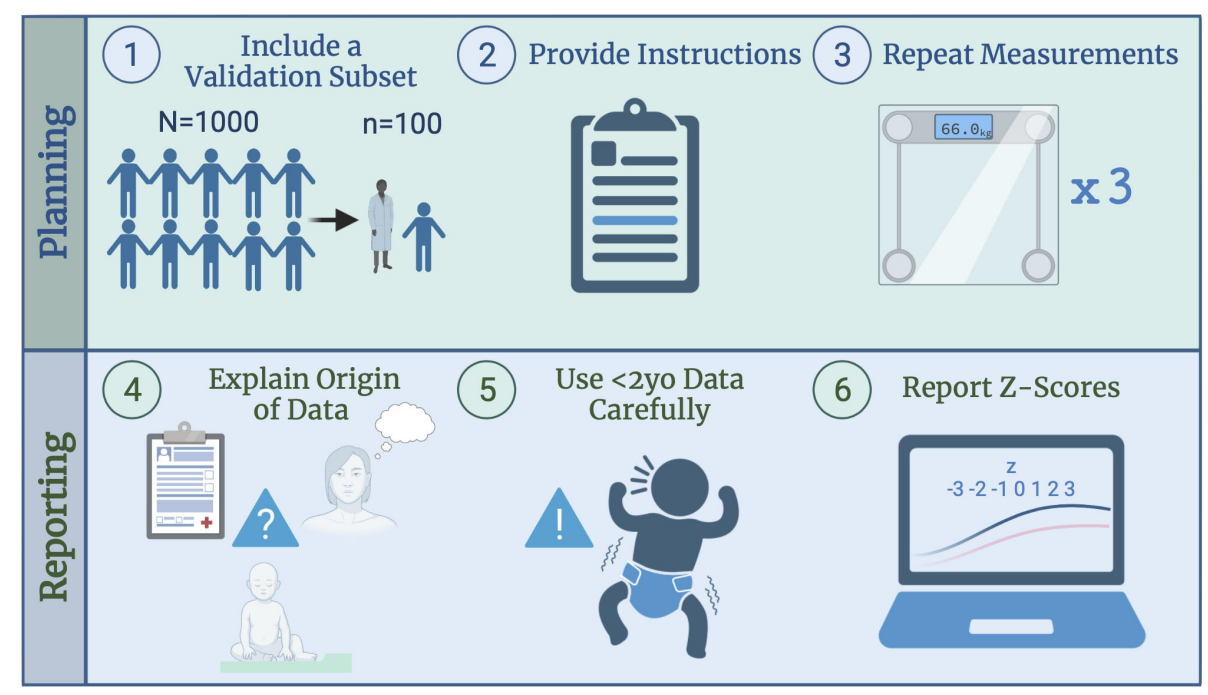

FIGURE 1 | Factors to consider when designing a study including remote, parent-reported infant length and weight measurements.

most logistically feasible method. Additionally, parent-reported measurements have value in their ability to promote participation and inclusion because participants need not live near clinical centers to participate. Remote anthropometry also enables the process of data collection for geographically dispersed and medically underserved populations who may not have access to or resources for in-person check-ups or care. Additionally, during infectious disease outbreaks, including the current pandemic, remote anthropometric measures can help reduce potential disease exposure by reducing time in clinic and facilitating remote health tracking through telemedicine $(17,18)$. For socially vulnerable families and for parents of children experiencing disease and disability, the benefits of remote anthropometry have the potential to outweigh the initially perceived and actual challenges, considering the likelihood for saving them time, money, and stress. These challenges include limited economic resources, lack of insurance, cultural barriers (language, mannerisms, social expectations), and health illiteracy, but can be partially overcome with virtual care strategies, according to the American Hospital Association (19).

\section{DISCUSSION}

Remote, parent-reported measurements of pediatric anthropometrics have been validated in children over 2 years old. With varying results, most show some degree of concordance between parent-reported and clinical metrics, especially in older age groups (20). However, there are several considerations both scientists and clinicians can make when conducting research or utilizing results that have incorporated parent-reported data (Figure 1).

\section{Planning Research}

1. Consider validating parent-reported measurements within a study by taking a representative sample of participants and comparing with specialist-obtained values. This can be especially useful if the population is geographically/ethnically/habitually unique, since existing validations are often based on populations that are not representatively diverse (20).

2. Provide parents/caregivers with a set of instructions regarding the desired method of anthropometric measurement. Instruct collection of measurements to the nearest tenth to limit heaping of parent-reported values at multiples of 0.5 , which limits accuracy (2). Consider providing parents of infants with instructions to make or obtain equipment necessary for accurate measurements (21), such as length boards or baby scales.

3. Have participants repeat measurements at each timepoint so that the average can be used for downstream analysis or clinical input (4). Data collected in triplicate will attenuate random error.

\section{Reporting Research}

4. Be clear in addressing the method by which child/infant caregivers derived the reported measurements (11)-i.e., Was it recall from a previous primary care visit? Was it an estimation? Was it measured by the parent themself? What type of instructions were provided?

5. Exercise caution when utilizing parent-reported measures of infant ( $<2$ years old) length and weight (22). The lack of validation studies in the existing literature is a gap that should be filled. Use remote measures as a back-up option if a patient population is inaccessible due to geography/circumstances, or quick nutritional/growth screening for rural populations is required (16).

6. For pediatric populations, report anthropometrics as a $\mathrm{z}$ score, since this method accounts for age and sex of the participants (2). Standalone height and weight values have 
limited analytical and interpretative value due to the highly variable growth rates and body sizes of infants and children.

\section{CONCLUSION}

Anthropometry is key to understanding the health of a population, and regarding children/infants, remote methods can be especially important for streamlining research, offsetting costs/burden, and improving accessibility to promote inclusion of diverse populations. As the field moves toward more widespread use of remote anthropometry, validation of these measurements is necessary to ensure they retain clinical and practical translatability.

\section{REFERENCES}

1. Casadei K, Kiel J. Anthropometric Measurement [Updated 2020 Apr 28]. Treasure Island, FL: StatPearls Publishing (2020). Available at: https://www. ncbi.nlm.nih.gov/books/NBK537315/ (accessed June 8, 2021).

2. Physical status: the use and interpretation of anthropometry. Report of a WHO Expert Committee. World Health Organ Tech Rep Ser. (1995) 854:1452. doi: 10.1002/(sici)1520-6300(1996)8:6<786::aid-ajhb11>3.0.co;2-i

3. Centers for Disease Control and Prevention. Anthropometry Procedures Manual. In: National Health and Nutrition Examination Survey (NHANES). (2007). p. 95. Available online at: http://www.cdc.gov/nchs/data/nhanes/ nhanes_07_08/manual_an.pdf

4. Viana Bagni U, da Silva Ribeiro KD, Soares Bezerra D, Cavalcante de. Barros D, de Magalhães Fittipaldi AL, Pimenta da Silva Araújo RG, et al. Anthropometric assessment in ambulatory nutrition amid the COVID-19 pandemic: possibilities for the remote and in-person care. Clin Nutr ESPEN. (2021) 41:186-92. doi: 10.1016/j.clnesp.2020.11.022

5. Hodge JM, Shah R, McCullough ML, Gapstur SM, Patel AV. Validation of selfreported height and weight in a large, nationwide cohort of US adults. PLoS ONE. (2020) 15:e0231229. doi: 10.1371/journal.pone.0231229

6. Tenenbaum A, Shefer-Averbuch N, Lazar L, Yakobovitch-Gavan M, Phillip M, Oron T. Growth assessment of children during the COVID-19 pandemicCan we rely on parental measurements? Acta Paediatr. (2021) 110:30405. doi: 10.1111/apa.16034

7. Scholtens S, Brunekreef B, Visscher TLS, Smit HA, Kerkhof M, Jongste JCD, et al. Reported versus measured body weight and height of 4-yearold children and the prevalence of overweight. Eur J Public Health. (2007) 17:369-74. doi: 10.1093/eurpub/ckl253

8. Brettschneider AK, Ellert U, Rosario AS. Comparison of BMI derived from parent-reported height and weight with measured values: results from the German KiGGS study. Int J Environ Res Public Health. (2012) 9:63247. doi: 10.3390/ijerph9020632

9. Chai LK, Collins CE, May C, Holder C, Burrows TL. Accuracy of parent-reported child height and weight and calculated body mass index compared with objectively measured anthropometrics: secondary analysis of a randomized controlled trial. J Med Internet Res. (2019) 21:e12532. doi: $10.2196 / 12532$

10. Huybrechts I, Beirlaen C, De Vriendt T, Slimani N, Pisa PT, Schouppe E, et al. Validity of instruction leaflets for parents to measure their child's weight and height at home: results obtained from a randomised controlled trial. BMJ Open. (2014) 4:e003768. doi: 10.1136/bmjopen-2013003768

11. Huybrechts I, Himes JH, Ottevaere C, De Vriendt T, De Keyzer W, Cox B, et al. Validity of parent-reported weight and height of preschool children measured at home or estimated without home measurement: a validation study. $B M C$ Pediatr. (2011) 11:63. doi: 10.1186/1471-2431-11-63

12. Conkle J, Keirsey K, Hughes A, Breiman J, Ramakrishnan U, Suchdev PS, et al. A collaborative, mixed-methods evaluation of a low-cost, handheld 3D imaging system for child anthropometry. Matern Child Nutr. (2019) 15:e12686. doi: $10.1111 / \mathrm{mcn} .12686$

\section{AUTHOR CONTRIBUTIONS}

EH contributed to conceptualization, investigation, visualization, and writing-original draft. TK contributed to validation and writing-review and editing. SC contributed to conceptualization, project administration, supervision, validation, and writing-review and editing. All authors have read and approved the final manuscript.

\section{ACKNOWLEDGMENTS}

Figure 1 was created with BioRender.

13. Yorkin M, Spaccarotella K, Martin-Biggers J, Quick V, Byrd-Bredbenner C. Accuracy and consistency of weights provided by home bathroom scales. BMC Public Health. (2013) 13:1194. doi: 10.1186/1471-2458-13-1194

14. Mansoor Y, Hale I. Parent perceptions of routine growth monitoring: A scoping review. Paediatr Child Health. (2021) 26:154. doi: $10.1093 / \mathrm{pch} /$ pxaa041

15. Humphrey G, Dobson R, Parag V, Hiemstra M, Howie S, Marsh S, et al. See How They Grow: Testing the feasibility of a mobile app to support parents' understanding of child growth charts. PLoS ONE. (2021) 16:e246045. doi: 10.1371/journal.pone.0246045

16. Weden MM, Brownell PB, Rendall MS, Lau C, Fernandes M, Nazarov Z. Parent-Reported Height and Weight as Sources of Bias in Survey Estimates of Childhood Obesity. Am J Epidemiol. (2013) 178:461. doi: 10.1093/aje/kws477

17. Alonso SG, Marques G, Barrachina I, Garcia-Zapirain B, Arambarri J, Salvador JC, et al. Telemedicine and e-Health research solutions in literature for combatting COVID-19: a systematic review. Health Technol (Berl). (2021) 11:1. doi: 10.1007/s12553-021-00529-7

18. Hollander JE, Carr BG. Virtually Perfect? Telemedicine for Covid-19 N Engl J Med. (2020) 382:1679-81. doi: 10.1056/NEJMp2003539

19. Bhatt J, Bathija P. Ensuring Access to Quality Health Care in Vulnerable Communities. Acad Med. (2018) 93:1271. doi: 10.1097/ACM.0000000000002254

20. O'Connor DP, Gugenheim JJ. Comparison of measured and parents' reported height and weight in children and adolescents. Obesity. (2011) 19:10406. doi: 10.1038/oby.2010.278

21. Yorkin M, Spaccarotella K, Martin-Biggers J, Lozada C, Hongu N, Quick V, et al. A tool to improve accuracy of parental measurements of preschool child height. Adv Public Heal. (2015) 2015:1-7. doi: 10.1155/2015/965371

22. Himes JH. Challenges of accurately measuring and using BMI and other indicators of obesity in children. Pediatrics. (2009) 124:s3-s22. doi: 10.1542/peds.2008-3586D

Conflict of Interest: The authors declare that the research was conducted in the absence of any commercial or financial relationships that could be construed as a potential conflict of interest.

Publisher's Note: All claims expressed in this article are solely those of the authors and do not necessarily represent those of their affiliated organizations, or those of the publisher, the editors and the reviewers. Any product that may be evaluated in this article, or claim that may be made by its manufacturer, is not guaranteed or endorsed by the publisher.

Copyright (c) 2022 Haddad, Kojaoghlanian and Comstock. This is an open-access article distributed under the terms of the Creative Commons Attribution License (CC $B Y)$. The use, distribution or reproduction in other forums is permitted, provided the original author(s) and the copyright owner(s) are credited and that the original publication in this journal is cited, in accordance with accepted academic practice. No use, distribution or reproduction is permitted which does not comply with these terms. 